\title{
Semiconductor optical fibres for infrared applications: A review
}

\author{
Anna C. Peacock ${ }^{1, *}$ and Noel Healy ${ }^{2}$ \\ ${ }^{1}$ Optoelectronics Research Centre, University of Southampton, Southampton, SO17 \\ 1BJ, UK \\ ${ }^{2}$ Emerging Technology and Materials Group, Newcastle University, NE1 7RU, UK \\ E-mail: * acp@orc.soton.ac.uk
}

\begin{abstract}
Over the last decade a new class of optical fibre has emerged that incorporates semiconductor materials within the core. These fibres are rich in optoelectronic functionality and offer extended transmission bands across the infrared spectral region so that their application potential is vast. Various fabrication methods have been developed to produce fibres with a range of unary and compound semiconductor core materials, which can be either amorphous or crystalline in form. This review discusses the main fabrication procedures and the infrared optical properties of the semiconductor fibres that have been fabricated to date, then takes a look at the future prospects of this exciting new technology.
\end{abstract}

Keywords: Semiconductor materials, optical fibres, fibre fabrication, semiconductor photonics, infrared optics

\section{Introduction}

Extending from the edge of the visible spectrum up to the millimeter wave regime, the infrared is a region of tremendous technological importance, with application areas that span from optical communications to imaging, spectroscopy, surgery and more $[1,2,3]$. This broad spectral band is usually separated into three categories, the near, mid and far-infrared. Although the precise definitions of these categories vary between disciplines, the emerging trend within the photonics community is to define the nearinfrared as $0.7-2 \mu \mathrm{m}$, the mid-infrared as $2-20 \mu \mathrm{m}$, and anything beyond this as the far-infrared [4]. In this context, the near-infrared wavelength range has been the most widely explored as it is home to the important telecommunications band. Consequently a large suite of low cost and efficient optical components are readily available for use in this region. This is particularly true for fibre components, of which silica-based glass fibres and fibre amplifiers are outstanding examples $[1,5]$. Together, these robust and flexible fibres form the backbone of our high-speed communications network, and 
thus have enabled the widespread access to information on which we are increasingly dependent [6].

Although no one would question the significant impact silica fibre components have had on our lives, they do suffer from a few key drawbacks. Firstly, they are somewhat limited in terms of their functionality, for example silica has low nonlinear optical coefficients and, being a dielectric, it has poor electronic properties [7]. Furthermore, they are only transparent up to wavelengths around $2.5 \mu \mathrm{m}$ and so cannot be used for longer mid to far-infrared applications [8]. Thus, faced with the increasing demands on photonic systems, there is growing interest in developing fibre components from new materials that can offer enhanced processing and transmission capabilities. In this regard, a wide range of materials and fibre designs have been considered for infrared waveguiding [9], with much focus being placed on those made from chalcogenide glasses owing to their favourable optical properties, but also because they can be fabricated via traditional drawing tower methods [10]. However, despite the fact that chalcogenide fibres have been around almost as long as their silica counterparts [11], so far their application has been limited due to mechanical and environmental stability issues [12].

In contrast, non-vitreous semiconductors, which includes the elemental (i.e., group IV) and compound (i.e., group II-VI and III-V) materials, not only exhibit exceptional optical and electronic properties, but they are also robust and stable even under conditions of high optical fluence [8]. As a result, in recent years a new class of fibre has emerged to incorporate crystalline and amorphous semiconductor core materials within traditional glass cladding structures $[13,14]$. Clearly, much of the motivation for these efforts stems from the enormous interest in semiconductor technologies [15] and the compelling prospect of exploiting the exceptional optoelectronic functionality of the materials directly within the glass fibre geometry. By unifying these two fundamental material platforms, semiconductor optical fibres have the potential to open up new possibilities in terms of integrated optoelectronic device development across an extended transmission range [16].

It is now ten years since the first semiconductor fibre fabrication method was reported, and several different approaches have since been developed that are capable of producing fibres with a range of core materials and waveguide designs $[17,18,19,20$, $21,22,23,24]$. The purpose of this Review is to highlight the advances that have been made in this area to date, and set the scene for the application of semiconductor fibres within the infrared spectral region.

\section{Semiconductor fibre fabrication}

There are two established fabrication methods for the production of semiconductor optical fibres, the high pressure chemical vapour deposition (HPCVD) technique and the molten core drawing (MCD) process. Both methods have their advantages and disadvantages, for example the HPCVD technique can be used to produce fibres with cores made from a large number of materials, but is limited to fibre lengths of a few 


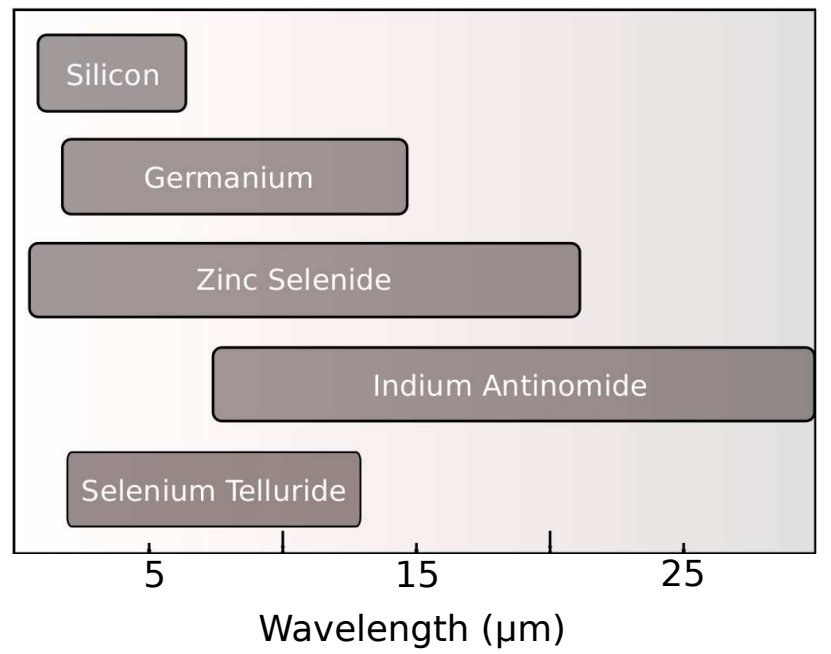

Figure 1. Transmission windows of some of the important infrared core materials used for the production of semiconductor optical fibres.

metres. Whereas the MCD process is restricted to materials that are compatible with the drawing process, but can yield kilometres of fibre. The techniques are very much complementary and both technologies have been used to produce a range of fibres with core materials suitable for infrared waveguiding, as shown in figure 1. In this section we review the two methods, highlighting the relative strengths and weaknesses of each.

\subsection{High pressure deposition}

In 2006 researchers at Pennsylvania State University and the Optoelectronics Research Centre at the University of Southampton produced the first crystalline semiconductor optical fibre [13]. In this instance, the team used a HPCVD process to grow elemental group IV materials inside the pores of silica micro-capillary templates. The capillaries have the dual function of acting as a reaction chamber for the deposition and as the low refractive index cladding material required for waveguiding. Prior to deposition, the precursors are mixed with a carrier gas to promote molecular transport though the capillary. The reaction is thermally initiated at the required location and, as the precursors decompose, the material is conformally deposited onto the capillary walls. Owing to the extremely smooth surfaces of the capillary templates (roughness values $\sigma \sim 0.1 \mathrm{~nm}[25]$ ) the core/cladding interface is near atomically smooth, which is particularly important for reducing scattering associated with the high index contrast, and thus achieving low loss waveguides [26]. The deposition is continued until the required film thickness is achieved or the pores are completely filled with material. The film thickness can be controlled to an accuracy of a few nanometers and it is possible to deposit different materials in concentric layers, thus providing an additional degree of freedom for fibre/device design.

By carefully choosing the precursors and deposition conditions, a large library of materials can be deposited including both unary and more complex semiconductors. 

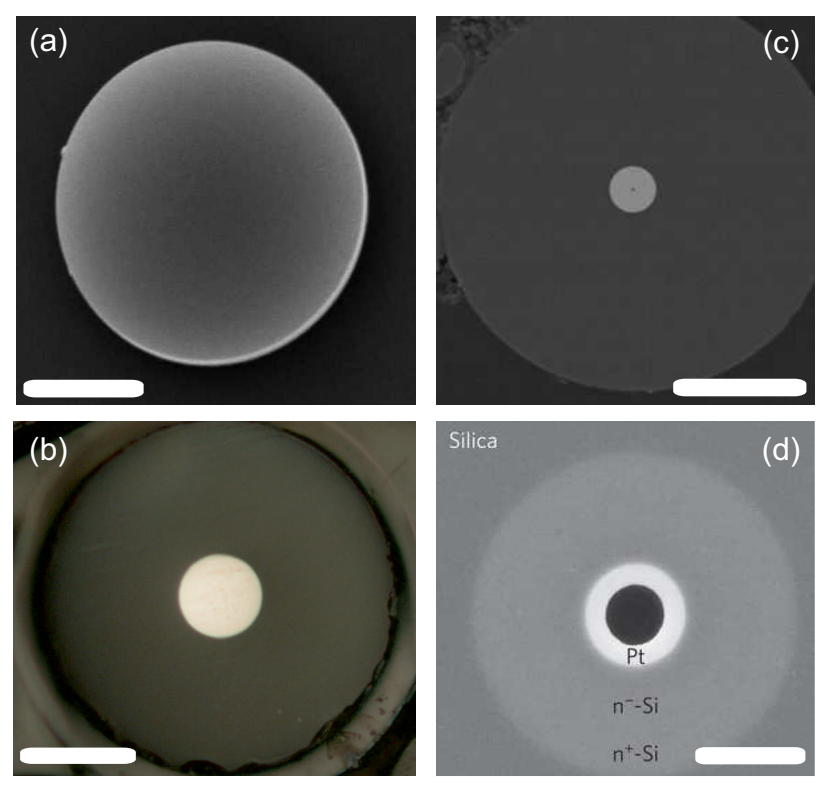

Figure 2. A selection of semiconductor optical fibres produced via the HPCVD method. (a) Silicon core optical fibre, scale bar $2 \mu \mathrm{m}$. (b) Germanium core optical fibre, scale bar $50 \mu \mathrm{m}$. (c) Zinc selenide core optical fibre, scale bar $40 \mu \mathrm{m}$. (d) In-fibre heterojunction, scale bar $2 \mu \mathrm{m}$.

Figure 2 displays a range of fibres that have been fabricated using the HPCVD technique. The silicon and germanium fibres shown in figure 2(a) and (b), respectively, have been completely filled. Such complete filling is possible when the reaction byproduct can diffuse out through the walls of the silica template [27]. However, there are situations where the byproduct is too large and must be exhausted through a nanoscale hole in the centre of the core [28], just visible for the zinc selenide (ZnSe) fibre of figure 2(c). Finally, figure 2(d) shows how the annular growth can be exploited for producing more complex core structures such as in-fibre heterojunctions [29].

A key advantage of the HPCVD method is that the temperatures and pressures can be controlled to fill capillary dimensions ranging from several tens of micrometres down to hundreds of nanometres. Furthermore, as the energy for precursor decomposition is significantly lower than that required for melting the bulk material, it can be undertaken at relatively low temperatures. This extends the available materials library beyond that of the MCD process to include materials that are amorphous, or that have particularly high melting temperatures. The ability to fabricate the fibres at low temperatures also means that materials that sublimate, or have thermal expansion coefficients that are mismatched with the cladding material, can be considered. However, there are also significant disadvantages associate with the HPCVD process. The most notable of these is the Arrhenius relationship between the deposition temperature and the reaction rate so that the depositions can be extremely slow, and for some materials the process can take a number of weeks to yield just a few centimeters of fibre. Another drawback is the great difficulty in growing large crystal grains in the micron-sized pores, and thus 


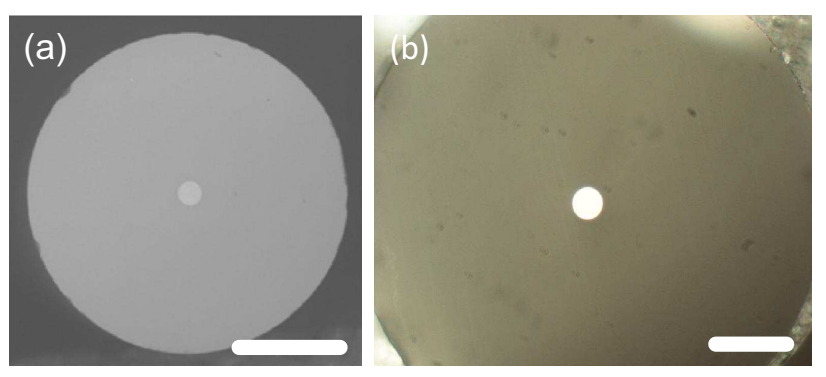

Figure 3. Silicon optical fibres produced via the MCD approach. (a) An early silicon fibre with a large core, scale bar $0.5 \mathrm{~mm}$. (b) A more recent fibre fabricated using a diffusion barrier to allow for micrometre core dimensions, scale bar $20 \mu \mathrm{m}$.

materials deposited directly in a polycrystalline form tend to suffer from high scattering losses.

\subsection{Molten core drawing}

The MCD technique is based on a modified fibre drawing method, whereby the glass cladding acts as a crucible that contains the semiconductor material as it is melted and drawn down to form the fiber core [30]. The process was first used to fabricate a semiconductor fibre by the Ballato group at Clemson University [14], but has since been widely adopted by a number of other research groups working in this area [20, 21, 22, 23]. Typically, the fibres are drawn from a preform consisting of a cladding glass tube that has been filled with the semiconductor material, which can be in the form of a solid rod or powder. The preform is then heated to above both the glass transition temperature of the cladding and the melting temperature of the semiconductor core. At this temperature the cladding glass can be deformed, but is still viscous enough to contain the molten semiconductor material and maintain its cylindrical geometry when drawn. As the fibre leaves the furnace, it rapidly cools and the liquid core solidifies into a polycrystalline form, which is surrounded by the glass cladding.

Key benefits of the MCD technique are that it can produce very long lengths of fibre and, by selecting the appropriate draw conditions, fibres with cores that consist of long (almost centimetre length) single crystal grains can be produced with relatively low optical losses [31, 32]. However, the high temperatures required to soften the cladding glass can introduce both positive and negative elements to the fabrication process. On one hand the high temperatures can be used to induce the reactive formation of semiconductor cores [22], but a direct consequence of this is that it can also result in significant diffusion of oxygen from the cladding into the core [14]. As well as limiting the minimum achievable core size, oxygen diffusion can also create unwanted inclusions and defects. Consequently, most of the early drawn semiconductor fibres where restricted to have large core dimensions, as shown in figure 3(a). An innovative approach to mitigate this issue is to introduce additives into the process that act as oxygen getters and/or diffusion barriers to isolate the core, so that fibres can now be produced with 
micrometre dimensions as shown in figure 3(b) [21,33]. Significantly, the introduction of diffusion barriers can also help to overcome another problem associated with the high temperatures, which is cracking and separation due to mismatches in the thermal expansion coefficients of the core and cladding materials. In this regard, there is also considerable ongoing work to identify alternative cladding glasses that may offer better compatibility with the core materials, both in terms of the draw temperatures and optical transparency [34].

\section{Infrared materials}

The optical transmission window for a solid material typically begins at a wavelength that corresponds to the electronic bandgap energy and ends at a wavelength associated with a vibrational resonance of the molecular structure. For example, the oxygen bond in oxide glasses limits their use to wavelengths below $2.5 \mu \mathrm{m}$. As a result, some of the most popular materials for infrared applications are based on materials with vibrational resonances at long wavelengths such as the halides, chalcogenides and semiconductors. Each family has its strengths and weaknesses, but owing to their high purity and excellent optoelectronic properties, semiconductors are beginning to excel for infrared photonics applications. Here we introduce the semiconductor materials that so far have been used in the fabrication of optical fibres. To facilitate the discussion, the materials are categorised into two groups, the single element unary semiconductors and the more complex multi-element compound semiconductors.

\subsection{Unary semiconductors}

3.1.1. Silicon Silicon is one of the most abundant materials on earth and is probably the most important material in the current technological epoch. It is no surprise that it is now being intensively studied for integrated photonics applications and that silicon photonics is a rapidly growing market [35]. In fact, the first proposal to use silicon as a photonics material was by Richard Soref in 1985 [36], but it has only been over the last decade that the fabrication processes have caught up with his vision. Silicon has many impressive optical properties such as a wide transparency window that spans from $1-7 \mu \mathrm{m}$, a high refractive index $n \sim 3.45$ that permits strong mode confinement, and a large third order optical nonlinearity of use for all-optical processing applications [15]. The near-infrared edge of this transmission window is defined by the electronic bandgap energy of $1.1 \mathrm{eV}$ and, as a result, silicon is transparent across the important telecommunications band. However, as this regime is relatively close to the band edge, two photon absorption (TPA) can be problematic at high optical intensities. Thus, as will be discussed in Section 4.3, for high power applications it is advantageous to work at longer infrared wavelengths $(>2 \mu \mathrm{m})$, where the sum of the two photon energies is no longer sufficient to span the bandgap. 

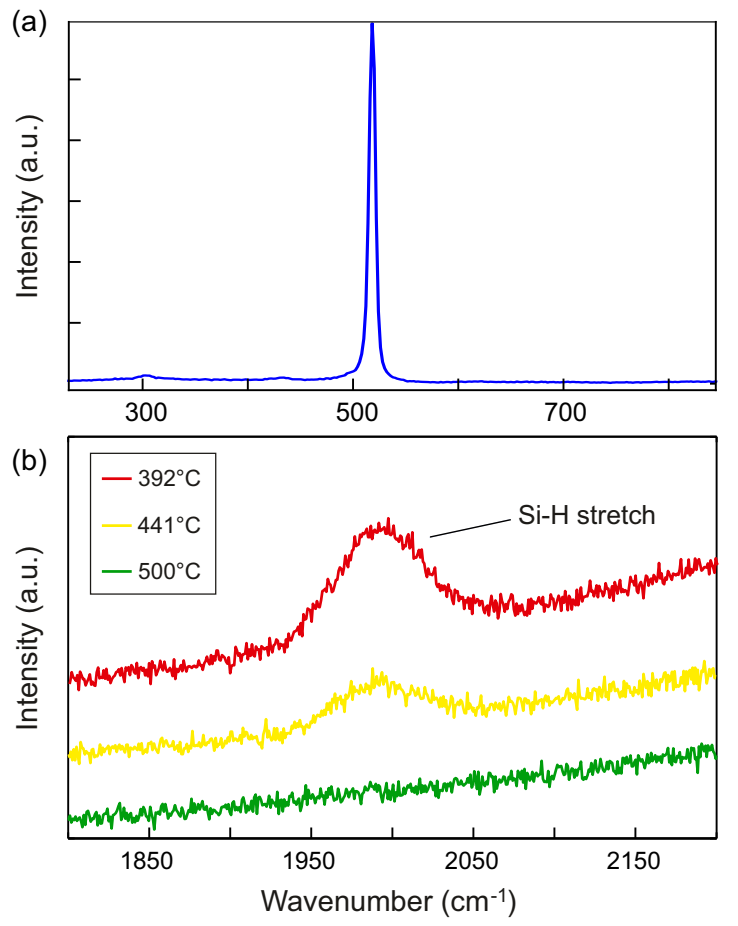

Figure 4. (a) Raman spectrum of a crystallised HPCVD silicon optical fibre. (b) Spectra for the Si-H vibration measured in a range of hydrogenated amorphous silicon optical fibres which can be used to provide a qualitative estimate of the hydrogen content as a function of deposition temperature.

Of all the semiconductor optical fibres, those with a silicon core have been the most widely studied and both the HPCVD and MCD techniques have been employed extensively to produce fibres of this type. As described in Section 2.2, the MCD approach always produces fibres with a polycrystalline core material. In contrast, fibres made via the HPCVD method are almost predominantly deposited in amorphous form, but it is straightforward to crystallize the core to polysilicon ( $\mathrm{p}-\mathrm{Si}$ ) post-fabrication via thermal annealing, as shown in figure 4(a). Interestingly, amorphous silicon (a-Si) is currently generating great interest in the optoelectronics community as it has a Kerr nonlinear coefficient, up to 5 times greater than crystalline silicon. On the downside, a-Si suffers from strong optical absorption due to the dangling bonds associated with its molecular disorder. However, if hydrogen is incorporated into the amorphous silicon (a-Si:H), it can passivate these bonds to significantly reduce the losses. This can be achieved using the HPCVD approach simply by restricting the dissociation of the precursor (silane $\mathrm{SiH}_{4}$ ) through reduced deposition temperatures [37], as shown in figure 4(b).

3.1.2. Germanium Although not as abundant as silicon, germanium has some clear advantages for infrared applications. For example, its optical transparency extends even further into the mid-infrared region, with a low loss transmission window from $2-15 \mu \mathrm{m}$. Thus, although the short wavelength edge precludes its use for telecommunications, germanium is transparent over all of the important wavelength windows for infrared 
sensing. It also has a higher refractive index than silicon $(n \sim 4)$ and a larger Kerr nonlinear coefficient (by $\sim 4$ times), so it has been predicted to be a useful material for the construction of long wavelength nonlinear optical devices [38]. Due to its relatively simple structure and its strong foundation in the electronics industry, germanium has received attention from proponents of both of the established semiconductor fibre fabrication methods. It is worth mentioning that as germanium's melting temperature $\left(\sim 940^{\circ} \mathrm{C}\right)$ is significantly lower than silicon $\left(\sim 1410^{\circ} \mathrm{C}\right)$, from a fabrication prospective, this presents advantages and disadvantages. For example, low processing temperatures can be beneficial for the drawn fibres as it allows for better matching of the core/cladding thermal expansion coefficients, but they can be problematic for the HPCVD method as it slows down the rate of deposition. As a result, both approaches favour the production of germanium fibres with smaller core dimensions, on the order of a few tens of microns or less.

\subsection{Compound semiconductors}

Though the increased molecular complexity of compound semiconductors make them more difficult to incorporate into the optical fibre geometry, there are some key rewards for doing so. For example, compound materials offer much more flexibility in terms of tuning their optoelectronic properties and many also have a direct electronic bandgap, thus opening a route to developing semiconductor in-fibre lasers. Furthermore, more complex crystal structures can provide a non-zero second order nonlinearity, which presents useful optoelectronic properties such as the electro-optic effect and the possibility for broadband second harmonic generation. Both the MCD and HPCVD methods have been used to produce fibres with important compound semiconductor core materials, as detailed below.

3.2.1. Zinc Selenide The II-VI compound semiconductor ZnSe is one of the most popular materials for the construction of optical components in the mid-infrared range. It has all of the advantageous properties mentioned above, including a very broad transmission window which spans from $0.5-22 \mu \mathrm{m}$, a large second order nonlinearity and is an excellent host for transition metal ions so that it can be used as a gain medium for mid-infrared lasers [39]. Unfortunately, ZnSe does not lend itself particularly well to the MCD technique as it sublimates at elevated temperatures, causing trapped gasses in the preform. In contrast, ZnSe fibres have been produced by the HPCVD method but, unlike silicon and germanium, this material is always deposited in a pure crystalline form, as evidenced by the Raman spectrum in figure 5(a). The main drawback of this fabrication approach is that complete filling of the capillary pores is difficult, as discussed in Section 2.1, though the remaining hole is typically less than $100 \mathrm{~nm}$. These holes are too small to be resolved using an optical microscope, but they can be imaged through the side of the fibre as its curvature acts as a lens, visible in figure 5(b). 


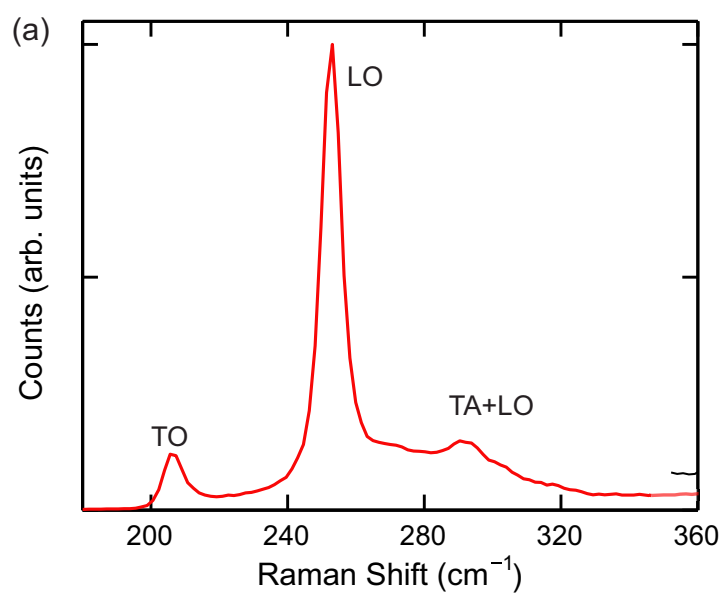

(b)

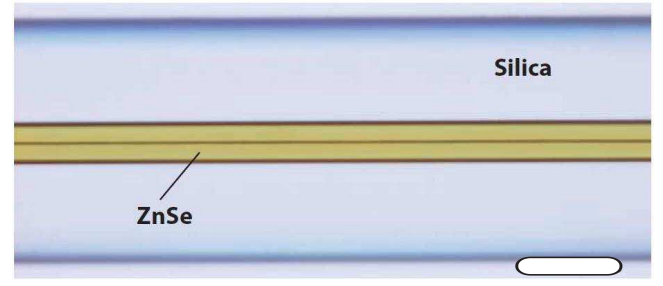

Figure 5. (a) The Raman spectrum of a zinc selenide optical fibre. (b) A diascopic micrograph of a zinc selenide optical fibre showing the incomplete filling of the core material, scale bar $50 \mu \mathrm{m}$.

3.2.2. Indium Antimonide The MCD fabrication method has also successfully produced compound semiconductor optical fibres and an excellent candidate for infrared applications is the group III-V core material indium antimonide (InSb) [17]. This material has a transmission window that spans from $7.3-30 \mu \mathrm{m}$, which is the longest infrared transmission edge of all the semiconductor optical fibres that have been developed to date (see figure 1). In contrast to the previous fibres, the InSb fibres were fabricated with a phosphate glass cladding which has a low glass transition temperature. This cladding was chosen both due to the low melting temperature of InSb, but also to better match the thermal expansion coefficients of the material. Unfortunately, even with this cladding there was significant diffusion of oxygen and phosphorous into the core during the drawing process, of substantial detrimental to the optical properties. However, the core material was shown to consist of large single crystal grains and thus, with the use of an appropriate diffusion barrier, high optical quality cores should be readily achievable.

3.2.3. Selenium Telluride The reactive molten core fabrication approach is an extension of the MCD technique whereby some chemistry is performed in the core during the high temperature drawing, so that the final material is quite different in composition 


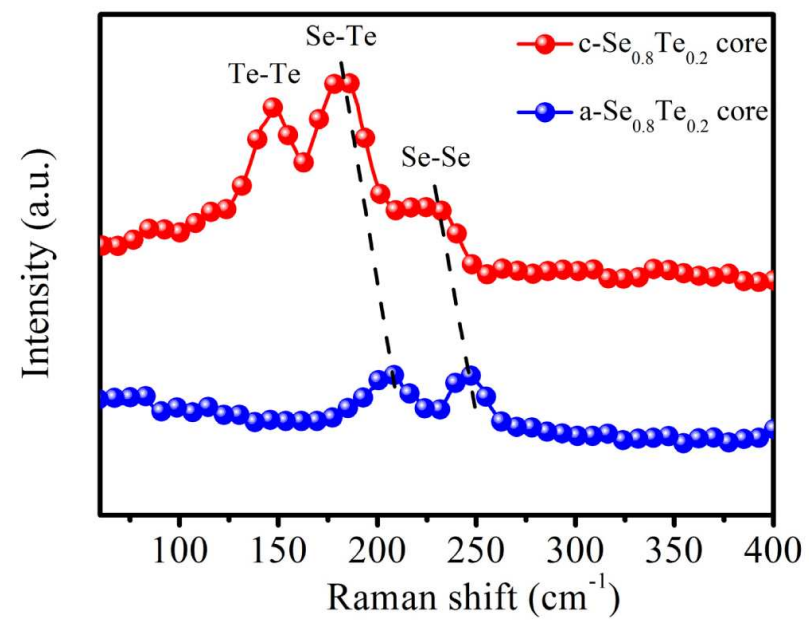

Figure 6. Raman spectra of the as-drawn selenium telluride optical fibre core (blue) and the annealed fibre core (red) [24]. Reprinted with the permission of the Optical Society of America.

from what it was in the preform. It was first used in the context of semiconductor core fibres by the Ballato group when they added silicon carbide to a silicon core to act as an oxygen getter, thus inhibiting oxygen diffusion from the cladding [33]. However, the approach is now finding more varied applications and a noteworthy example is the production of SeTe alloy core fibres [24]. SeTe semiconductors have rich optoelectronic properties such as photoconductivity, high optical nonlinearities and a large infrared transparency ranging from $2-12 \mu \mathrm{m}$. Similar to the InSb fibres, the cladding material used to produce the SeTe fibre preform was a phosphate-based glass, which was filled with a mixture of Se and Te powder (4:1 molar ratio). The drawn fibre had a uniform core composition of $\mathrm{Se}_{0.8} \mathrm{Te}_{0.2}$, but the crystallinity was relatively poor. As illustrated in figure 6 , subsequent annealling was shown to dramatically improve the crystallinity and, with further optimization of the post-processing, this method is expected to be very useful for the production of high optical quality alloy fibres.

\section{Optical properties}

The possibility to fabricate a wide assortment of fibres consisting of various semiconductor core materials and design compositions opens up many new application areas. In this section we discuss the transmission properties of a selection of fibres that have been fabricated to have promising optical properties across the infrared spectral region.

\subsection{Transmission losses}

The simplest fibre design is the 'step-index' structure, which consists of a high index core surrounded by a lower index cladding. These fibres guide light via total internal 
reflection, with the waveguiding parameters determined by the core size and the index contrast. Here we present the results of linear transmission measurements for stepindex fibres with the core materials discussed in Section 3. Typically, the loss values are measured via the cut-back method using fibres of only a few centimetres in length. As the guided light is highly confined to the core, these loss values are a good indication of the semiconductor material quality.

4.1.1. Silicon Fibres Figure 7(a) shows representative loss measurements for both amorphous and crystalline silicon fibres fabricated via the HPCVD and MCD methods. Perhaps somewhat surprisingly, at the time of this Review, the lowest loss value reported in a silicon fibre was measured in an a-Si:H core material $(\sim 0.3 \mathrm{~dB} / \mathrm{cm}$ at a wavelength of $2.7 \mu \mathrm{m}$ ) [40]. The low losses that have been obtained in these fibres is a result of continued efforts to control the concentration and distribution of hydrogen throughout the core during the deposition method [27]. However, the loss values for the p-Si fibres fabricated by the MCD method are not too far behind, most likely due to the large crystal grains that are produced via the drawing method [14, 21, 32]. Further improvements to the p-Si core materials can be made post-process using thermal [41, 42] or laser-induced annealing treatments [43] and in fact, the lowest losses reported for the MCD fibres were obtained after processing with a $\mathrm{CO}_{2}$ laser [44].

4.1.2. Germanium Fibres Figure 7(b) presents the lowest loss values that have been reported for germanium core fibres. For the HPCVD method the fibre has an amorphous core (a-Ge) and for the MCD approach the core was polycrystalline (p-Ge). Like the a-Si fibres, the key to reducing the losses in the a-Ge fibres is the incorporation of hydrogen to passivate the dangling bonds. However, as the Ge-H bond is much weaker than its $\mathrm{Si}-\mathrm{H}$ counterpart, it is more difficult to capture sufficient hydrogen to obtain such low losses [45]. Thus, by far the lowest loss that has been reported for the germanium fibres was measured in the MCD p-Ge core fibre $(\sim 1 \mathrm{~dB} / \mathrm{cm}$ at $3.4 \mu \mathrm{m})$ [31], and it is likely that this could be decreased further via laser annealing.

4.1.3. Compound Semiconductor Fibres As mentioned in Section 3.2, the list of compound semiconductor core materials includes InSb, ZnSe, and SeTe alloys in both amorphous $\left(\mathrm{a}-\mathrm{Se}_{0.8} \mathrm{Te}_{0.2}\right)$ and crystalline $\left(\mathrm{c}-\mathrm{Se}_{0.8} \mathrm{Te}_{0.2}\right)$ forms. As yet, no transmission measurements have been conducted in the InSb and $c-\mathrm{Se}_{0.8} \mathrm{Te}_{0.2}$ fibres, owing to the diffusion and crystallinity issues that were discussed previously. Thus figure 7(c) only reports loss values for the $\mathrm{ZnSe}$ and $\mathrm{a}-\mathrm{Se}_{0.8} \mathrm{Te}_{0.2}$ core fibres. Significantly, the losses measured for the ZnSe fibres beyond $2 \mu \mathrm{m}$ are the lowest obtained in a polycrystalline semiconductor core fibre to date, which has been attributed to the very high material quality of the crystal grains. Similarly, the loss value for the a-Se ${ }_{0.8} \mathrm{Te}_{0.2}$ fibre of $\sim 2 \mathrm{~dB} / \mathrm{cm}$ at $1.55 \mu \mathrm{m}$ is also very reasonable. Thus it is hoped that with further optimization of the compound and alloy core materials these fibres could be used for the 

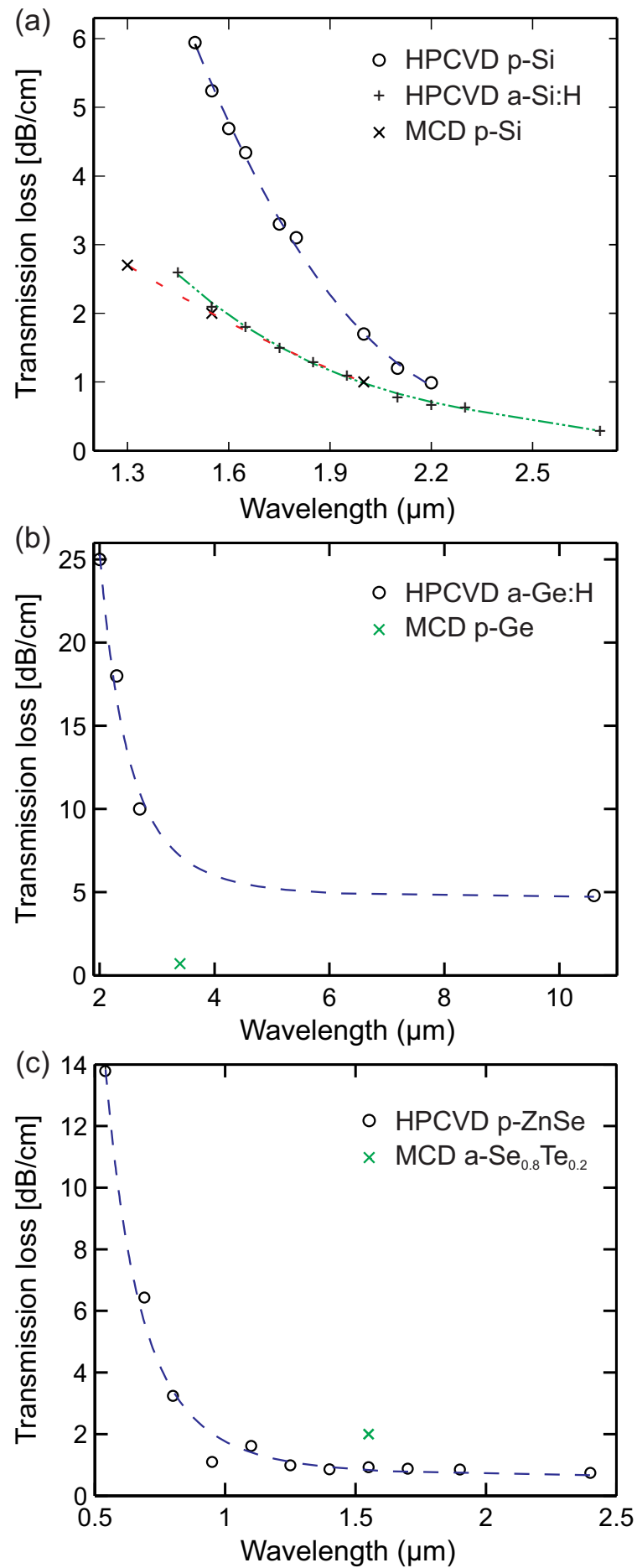

Figure 7. Representative transmission losses for a selection of semiconductor stepindex fibres. (a) Silicon core fibres, (b) germanium core fibres, and (c) semiconductor compound and alloy core fibres. The values are taken from the references: $[14,24,28$, $31,40,42,44,45]$.

development of active optoelectronic devices across the near to mid-infrared transmission bands, as will be discussed in Section 5.2. 

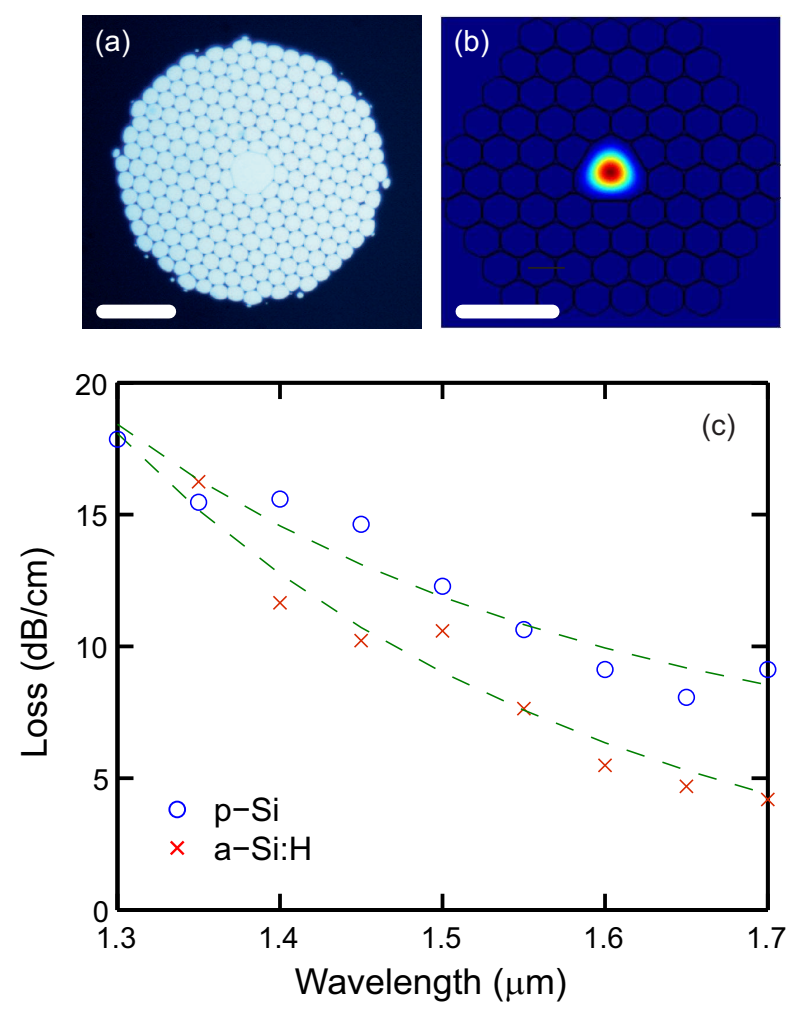

Figure 8. (a) Silicon microstructured fibre fabricated via complete filling of a hollowcore silica MOF, scale bar $15 \mu \mathrm{m}$. (b) Modelled fundamental mode profile at $1.55 \mu \mathrm{m}$, scale bar $10 \mu \mathrm{m}$. (c) Transmission losses for MOFs filled with p-Si and a-Si:H materials, see legend.

\subsection{Novel designs}

A key advantage of the semiconductor fibre platform is that it is possible to exploit novel waveguide designs and device geometries that are not readily accessible in planar forms. Two notable examples that allow for strong tailoring of the mode properties are the microstructured optical fibres (MOFs) and fibre-based resonators described below.

4.2.1. Semiconductor MOFs One of the problems of the step-index design is that the high index contrast between the semiconductor cores and the glass cladding materials means that these fibres typically support a large number of guided modes [46]. One possible solution to reducing the number of modes is simply to reduce the core size [47], but this comes at the expense of reduced coupling efficiency, particularly when coupling to conventional glass fibres. An alternative approach is to make use of two-dimensional microstructured fibres that can be carefully designed to control the modal properties of the core [48]. For example, figure 8(a) shows a silicon MOF that has been designed to have a reduced core/cladding effective index contrast by incorporating silicon microwires into the cladding [49]. These high index cladding inclusions act to disrupt the guidance of the higher order core modes so that it is possible to selectively couple into the lowest order modes. Thus, despite the large $9 \mu \mathrm{m}$ core diameter, investigations of the mode 
properties show that this fibre design principally supports the two lowest order core modes, with the fundamental mode profile shown figure $8(\mathrm{~b})$. Owing to the complexity of the design, such silicon MOFs are best suited to the HPCVD fabrication method, and thus the materials can be either amorphous or polycrystalline in nature. The corresponding losses for fibres fabricated from both materials are shown in figure 8(c), where the values for the a-Si:H core fibre are lower, in accordance with the materials in figure 7(a). Although the losses measured for the silicon MOFs are slightly higher than the step-index results, with continued improvements to the fibre design and materials it should be possible to fabricate high quality large core silicon fibres with essentially single mode guidance [50]. If these fabrication hurdles can be overcome, then we expect the MOF designs to be of particular use for long wavelength infrared applications, both due to the larger cores and because of the reduced content of silica in the cladding. It is also worth noting that this approach can be easily extended to the other semiconductor materials compatible with the HPCVD fabrication method and, for example, germanium and ZnSe MOFs have also been demonstrated [28, 51].

\subsubsection{Semiconductor fibre resonators Another way to tailor the mode confinement in} the semiconductor core fibres is to turn them into whispering gallery mode (WGM) micro-resonators. In this configuration, instead of propagating longitudinally down the fibre, the modes are confined to circulate around the axis, confined by total internal reflection at the outer surface [52]. Although for silica fibres the outer surface of the resonator can simply be defined by the cladding, in the case of the semiconductor fibres it is necessary to first etch away the cladding glass so that the light can be coupled directly into the high index core. An example of an etched silicon fibre micro-resonator is displayed in the inset of figure 9(a). An important feature of these structures is that, as mentioned in Section 2.1, the semiconductor core material has a negligible surface roughness defined by the pristine glass cladding [53]. As surface scattering is one of the dominant loss mechanisms in WGM resonators, ultra-smooth surfaces are critical for a obtaining high quality $(Q)$ factor, i.e. low loss, resonators. Figure 9(a) shows a typical transmission spectrum measured for a $6 \mu \mathrm{m}$ diameter a-Si:H fibre resonator when launching into the WGMs via a standard tapered silica fibre coupler approach [52]. This spectrum reveals $Q$-factors on the order of $10^{4}$, which are comparable to the best values reported in planar-based single crystal silicon resonators [54], and these could be improved further by optimizing the material and coupling conditions.

Significantly, as these WGM resonators are essentially free-standing, for appropriate choices of the starting fibre core diameter they are capable of operating across the full infrared transmission window of the semiconductor material. Thus fibre-based resonators that are fabricated from ZnSe materials can potentially operate from the visible up to the far-infrared. Figure 9(b) shows a typical transmission spectrum for a ZnSe WGM resonator fabricated from a fibre with a $15 \mu \mathrm{m}$ diameter core (see inset), from which we obtain $Q \sim 10^{4}[55]$. The fact that the $Q$-factors are comparable to the silicon fibre resonators is not surprising as the surface roughnesses is dictated by 

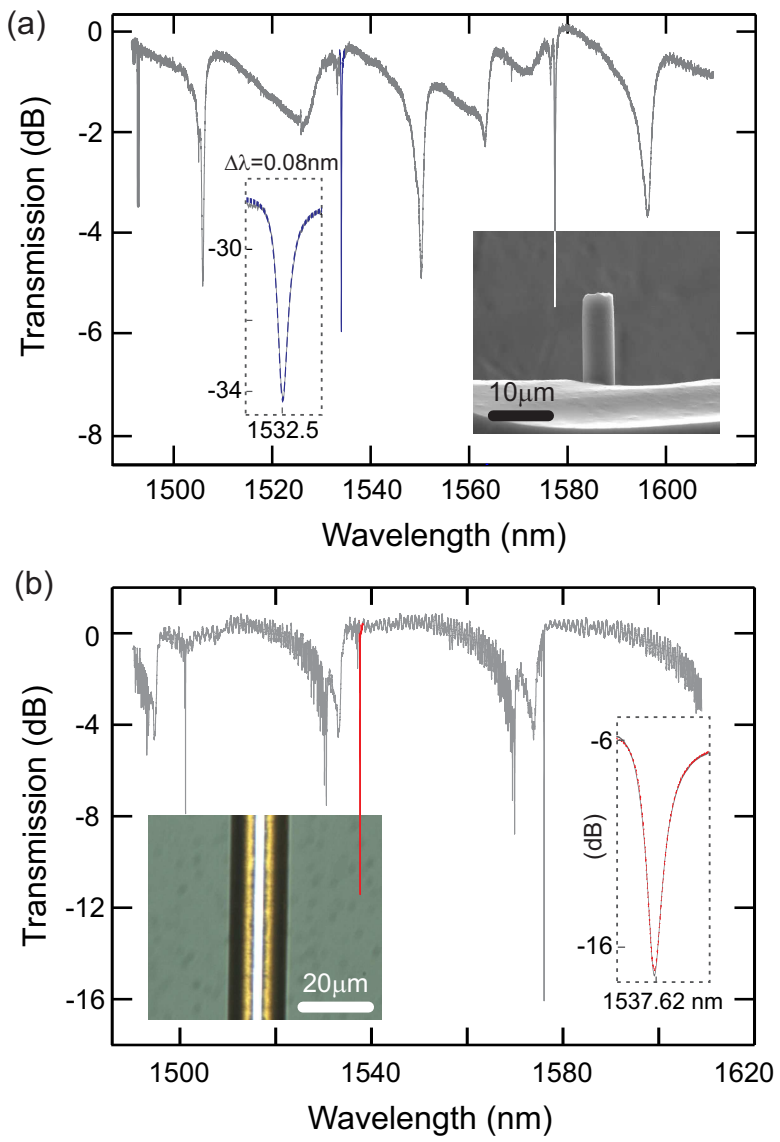

Figure 9. Transmission spectra for fibre-based resonators fabricated from (a) an a$\mathrm{Si}: \mathrm{H}$ core and (b) a ZnSe core. In both cases the insets show the etched core and a fit to determine the $Q$-factor of the highlighted resonance.

the cladding (which in both cases is silica) and the losses are also well-matched around $1.55 \mu \mathrm{m}$ (see figure 7). It is worth noting that although the characterizations so far have been conducted within the near-infrared spectral band, this is simply due to the lack of sources and diagnostics available at the longer wavelengths and the lower losses obtained for both materials in the mid-infrared suggest that the resonators should perform well in this region.

\subsection{Nonlinear effects}

There are several key parameters that must be considered when selecting an appropriate optical fibre for nonlinear propagation such as transmission loss, length, core size and material [56]. Obviously these parameters can be traded against each other, for example if the losses are high then the core should be relatively small to increase the mode intensity, and if the core is small then typically the length does not need to be so long [57]. Furthermore, owing to the high nonlinear coefficients of the semiconductor materials when compared to silica (up to several orders of magnitude), the required fibre lengths can be considerably shorter and the losses much larger for comparable core 

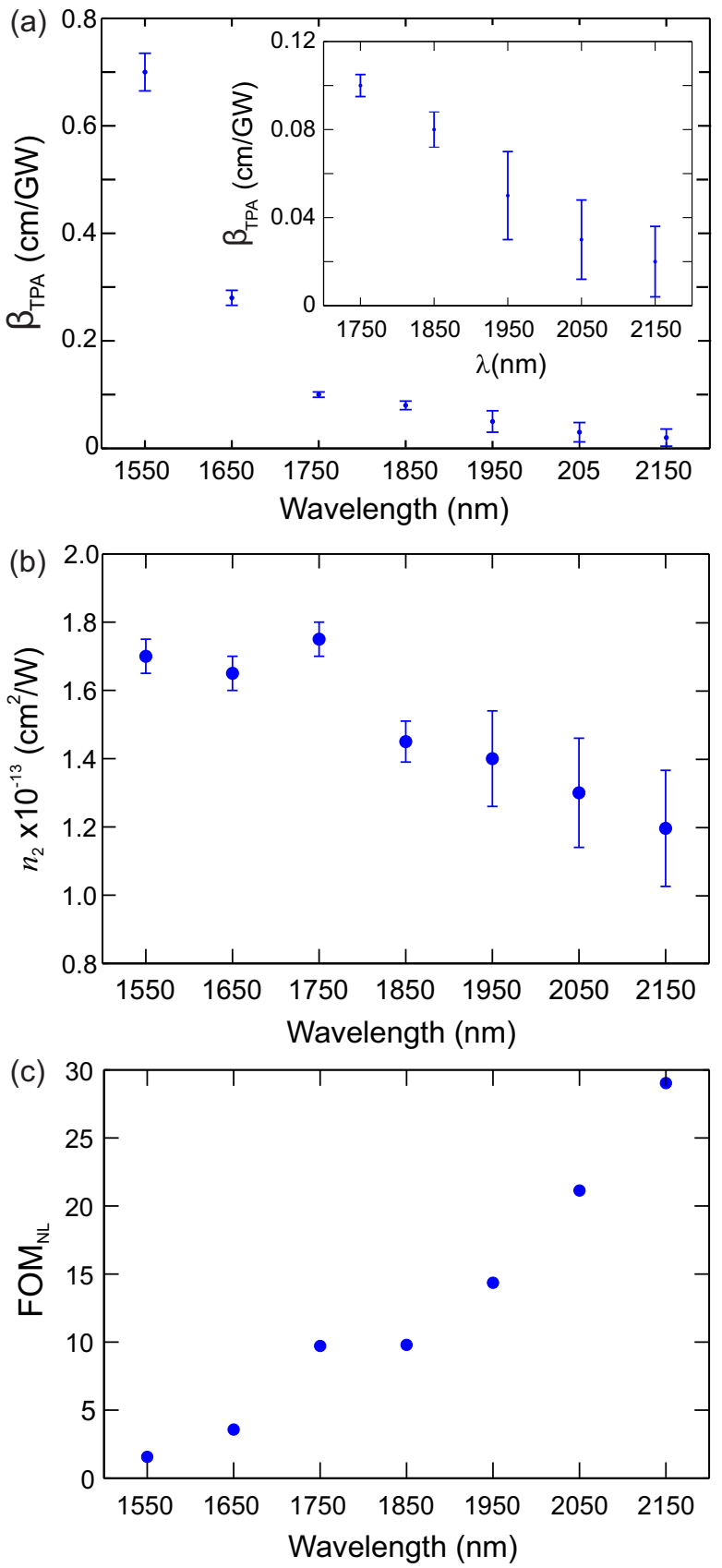

Figure 10. (a) TPA parameter (inset shows a close up of the long wavelength region) and (b) Kerr nonlinear index as functions of wavelength. The error bars represent the uncertainty in the input powers. (c) Corresponding nonlinear figure of merit.

sizes [58]. Taking this into consideration, out of all the semiconductor fibres fabricated to date, the a-Si:H core fibres have been the most suited for nonlinear investigations as the material has a high nonlinearity and the fibres can be produced to have relatively low losses $(\sim 1 \mathrm{~dB} / \mathrm{cm})$, small core sizes of a few micrometres in diameter $(2-6 \mu \mathrm{m})$, and lengths of several centimetres [59].

As a-Si:H is a uniform symmetric material, nonlinear transmission in these fibres is governed by processes involving the third order $\left(\chi^{(3)}\right)$ nonlinearity. The real part 
of $\chi^{(3)}$ gives rise to Kerr nonlinear refraction and the imaginary part to TPA. The easiest nonlinear effect to observe is TPA, which manifests as an intensity dependent absorption. Thus the magnitude of the TPA parameter $\left(\beta_{\mathrm{TPA}}\right)$ can be determined by simply monitoring the transmitted power through the fibre as a function of the coupled input power [58]. Although nonlinear absorption is undesirable in many high power applications, the ultrafast nature of TPA can be exploited to realize all-optical processing functions such as pulse shapers, logic gates, modulators, switches, and detectors [60]. Figure 10(a) shows the measured values for $\beta_{\text {TPA }}$ for the a-Si:H fibres across the nearinfrared and up to the edge of the mid-infrared [40]. These results show that $\beta_{\text {TPA }}$ initially drops sharply across the telecoms wavelength range of $1.55-1.65 \mu \mathrm{m}$, then begins to plateau, eventually approaching negligible values for $\lambda>2 \mu \mathrm{m}$. This behaviour is as we would expect as the bandgap of a-Si:H is $E_{g} \sim 1.5 \mathrm{eV}$, and so when the photon energy goes below $E_{g} / 2$ the sum of two photons is no longer sufficient to span the bandgap.

The second effect of Kerr refraction gives rise to an intensity dependent phase shift across the propagating pulse, which manifests as spectral broadening. Thus the size of the nonlinear refractive index $\left(n_{2}\right)$ is determined by monitoring the spectral components of the pulse at the output of the fibre [58], and figure 10(b) shows the values obtained for the a-Si:H fibre across the same wavelength range as figure 10(a) [40]. The trend seen in these results, where the $n_{2}$ values increase near $E_{g} / 2$ before dropping off for longer wavelengths, is in agreement with the nonlinear Kramers-Kronig relation [61]. However, although one might expect that the largest values of $n_{2}$ will give rise to the greatest broadening, as a strong TPA parameter reduces the pulse intensity, there is a trade off between these two parameters. This can be quantified through a nonlinear figure of merit: $\mathrm{FOM}_{\mathrm{NL}}=n_{2} / \beta_{\mathrm{TPA}} \lambda$. The corresponding $\mathrm{FOM}_{\mathrm{NL}}$ for these a-Si:H fibres is shown in figure 10(c), from which it is clear that the Kerr effect can actually be more efficient for wavelengths $>2 \mu \mathrm{m}$. Thus these results suggest that the silicon core fibres will perform better for nonlinear applications in the mid-infrared wavelength regime, significantly where the performance of traditional silica fibres starts to degrade.

As a means to benchmark the nonlinear performance of the a-Si:H fibres, a number of basic functions have been demonstrated including all-optical modulation and wavelength conversion in the telecoms band [59, 62], and supercontinuum generation out to the mid-infrared [63]. Although the efficiency of the nonlinear processes in these fibres are comparable to some of the best demonstrations in planar waveguides $[64,65]$, the larger core sizes necessitate the use of higher power levels. However, a simple way to reduce the power requirements is to make use of the resonator geometry to enhance the light-matter interactions, as has been illustrated using an optical modulation experiment where the coupled pump power into the WGMs could be reduced by four orders of magnitude when compared to the standard transmission mode [66]. It is also worth mentioning that owing to the recent improvements in the quality of the polycrystalline core materials, nonlinear demonstrations in these fibres are now starting to emerge. This includes the first demonstration of nonlinear propagation in a p-Si core fibre [67], 
as well as second harmonic generation in a ZnSe fibre resonator [55]. The latter result is particularly significant as this was the first time that a fibre with a large, intrinsic second order nonlinearity has been used for efficient broadband wavelength conversion. Furthermore, by exploiting the unique phase-match conditions offered by the cylindrical resonators, it should be possible to excite more complex nonlinear processes such as sum and difference-frequency generation, opening a route for wavelength conversion deep into the infrared.

\section{Perspectives and outlook for future devices}

The optical characteristics of the semiconductor core fibres presented in this article clearly highlight their potential for use in passive and active devices in the infrared. Although the platform is still quite young, it is developing at an extraordinary rate and a number of important devices have already been demonstrated with performances that rival their planar counterparts $[66,67]$. However, clearly there are still several challenges to overcome in terms of optimizing the existing materials as well as extending the library, which will ultimately lead to enhanced device functionality in areas such as all-optical signal processing, sensing, medicine, light generation and amplification.

\subsection{Sensors}

The mid-infrared is home to a number of spectral 'fingerprint' regions that are important for optical sensing of biological and chemical analytes. This is one of the key drivers for the rapid increase in the development of sources and materials in this spectral region. Sensors based on waveguides are of great interest for applications in environmental monitoring, security, and the biomedical industry as they can offer high efficiencies and are also very robust. Indeed, this is one of the key advantages accredited to the lab-on-a-chip architecture, which is now rigorously exploring silicon and germanium as platform materials. Thus we anticipate that the semiconductor fibres will similarly have an important role to play in the future of mid-infrared sensing. In particular, these fibres could act as the optical source, delivery mechanism, and even the actuators for a broad range of measurands over an extended wavelength range. Furthermore, as the micro-resonator configurations described in Section 4.2.2 have resonant frequencies that are highly sensitive to index modulation, they could be used to detect mass loading and temperature changes induced by chemical reactions with astounding sensitivity [68].

\subsection{Amplifiers and lasers}

Semiconductors have a long history as gain media for lasers and amplifiers and the semiconductor diode is the most prevalent laser type in use today. These devices are typically bulk or planar-based structures that are electrically pumped. However, the possibility to construct semiconductor lasers within a fibre geometry presents many advantages as the devices would be robust, stable, have excellent beam quality, 
require minimal alignment, and the power scaling would be straightforward as thermal management is easier over longer lengths.

The simplest form of a semiconductor fibre amplifier or laser would be to exploit the large Raman gain coefficient of the group IV core materials and make use of an optical pumping scheme. In this regard, silicon has already been demonstrated for amplification and lasing using a planar silicon waveguide [69]. Clearly, one of the key benefits of developing an in-fibre silicon Raman amplifier, and/or laser, is that it could be easily integrated with existing fibre infrastructures, including the high power pump sources. Furthermore, as Raman gain is achievable at any wavelength where there is a suitable pump source, this opens a route to developing amplifiers and lasers across the entire infrared transmission band of the silicon, or indeed germanium, core fibres.

An alternative type of optically pumped semiconductor light source would be to make use of the direct bandgap compound semiconductors doped with transition metals. For example, ZnSe can be doped with either chromium or iron ions to produce efficient gain media in the $2-3 \mu \mathrm{m}$ and $4-5 \mu \mathrm{m}$ regions, respectively [39]. As the HPCVD process can facilitate controlled doping, further progress in this area is expected to produce semiconductor optical fibres that can function as active gain elements for a new generation of mid-infrared optical fibre amplifiers and lasers.

\subsection{Medical probes}

The infrared is of great relevance for medical applications and there is a high demand for lasers that operate in this region that can be used for illumination, imaging, and cutting soft tissue. However, as each new laser emerges, so does the need for optical fibres that can deliver, and/or collect, light from around the human body. Recent examples where the semiconductor fibres could find potential use include near-infrared imaging of brain tissue [70] and mid-infrared imaging of tissues and biofluids [71]. Significantly, by exploiting the ultra-broad transmission windows of the semiconductor core materials, it will be possible to simultaneously guide several laser sources within a single fibre, so that they could be used to diagnose, analyse and administer treatment in the one intervention. Furthermore, these fibres also offer high damage thresholds, are biocompatible, and are non-toxic, all of which are critical for surgical applications.

\section{Conclusion}

The field of infrared fibres is currently experiencing a dramatic growth, with many new materials, waveguide designs and fabrication techniques being investigated. The semiconductor fibre platform described in this Review represents only a subset of these, and there are a number of high quality reviews in the literature that are relevant to this topic area $[72,73,74,75,76]$. However, as we have outlined, there are a wide range of semiconductor materials that are compatible with the fibre fabrication methods and, as well as offering extended transparency windows, these materials are rich in 
optoelectronic functionality. Owing to the early stage in the development of these fibres most of the characterization and device development has focused on the nearinfrared, though, with continued reduction in transmission losses, we expect that their use will rapidly extend into the mid and eventually far-infrared spectral regions. In fact, reducing the loss values towards the $\sim 1 \mathrm{~dB} / \mathrm{m}$ level remains a critical challenge in terms of improving the practicality of the semiconductor fibre technology, and should be a key target for the community over the next decade. Once this milestone has been achieved, it is easy to predict that semiconductor fibres will play an important role within a host of infrared application areas, as well as open the door to many new and exciting possibilities.

\section{Acknowledgements}

The authors acknowledge their colleagues and students who have provided valuable contributions to the cited work, particularly P. Mehta, L. Shen, F. Suhailin, L. Xiao, N. Vukovic, J. Sparks, T. Day, J. Badding, U. Gibson and J. Ballato. Financial support has been provided by EPSRC (EP/G051755/1 and EP/J004863/1). The data presented in this article is available from the corresponding author on request.

\section{References}

[1] S. E. Miller, E. A. J. Marcatili, and T. Li. Research toward optical-fiber transmission systems. Proc. IEEE, 61:1703, 1973.

[2] O. S. Wolfbeis. Fiber-optic chemical sensors and biosensors. Anal. Chem., 74:2663, 2002.

[3] B. Temelkuran, S. D. Hart, G. Benoit, J. D. Joannopoulos, and Y. Fink. Wavelength-scalable hollow optical fibres with large photonic bandgaps for $\mathrm{CO}_{2}$ laser transmission. Nature, 420:650, 2002.

[4] Extending opportunities. Nat. Photon., 6:407, 2012.

[5] R. J. Mears, L. Reekie, I. M. Jauncey, and D. N. Payne. Low-noise erbium-doped fibre amplifier operating at $1.54 \mu \mathrm{m}$. Electron. Lett., 23:1026, 1987.

[6] R. J. Essiambre and R. W. Tkach. Capacity trends and limits of optical communication networks. Proc. IEEE, 100:1035, 2012.

[7] G. P. Agrawal. Fiber-Optic Communication Systems, 4th Edition. Wiley, 2010.

[8] M. Bass. Handbook of Optics: Volume IV - Optical Properties of Materials, Nonlinear Optics, Quantum Optics, Third Edition, chapter 5. McGraw-Hill Professional, 2010.

[9] J. A. Harrington. Infrared Fibers and Their Applications. SPIE PRESS, 2004.

[10] J. S. Sanghera and I. D. Aggarwal. Active and passive chalcogenide glass optical fibers for IR applications: a review. J. Non-Cryst. Solids, 256:6, 1999.

[11] N. S. Kapany and R. J. Simms. Recent developments in infrared fiber optics. Infrared Physics, $5: 69,1965$.

[12] B. J. Eggleton, B. Luther-Davies, and K. Richardson. Chalcogenide photonics. Nat. Photon., 5:141, 2011.

[13] P. J. A. Sazio, A. Amezcua-Correa, C. E. Finlayson, J. R. Hayes, T. J. Scheidemantel, N. F. Baril, B. R. Jackson, D.-J. Won, F. Zhang, E. R. Margine, V. Gopalan, V. H. Crespi, and J. V. Badding. Microstructured optical fibers as high-pressure microfluidic reactors. Science, 311:1583, 2006. 
[14] J. Ballato, T. Hawkins, P. Foy, R. Stolen, B. Kokuoz, M. Ellison, C. McMillen, J. Reppert, A. M. Rao, M. Daw, S. Sharma, R. Shori, O. Stafsudd, R. R. Rice, and D. R. Powers. Silicon optical fiber. Opt. Express, 16:18675, 2008.

[15] B. Jalali and S. Fathpour. Silicon photonics. J. Lightwave Technol., 24:4600, 2006.

[16] D. Graham-Rowe. Fibres get functional. Nat. Photon., 5:66, 2011.

[17] J. Ballato, T. Hawkins, P. Foy, C. McMillen, L. Burka, J. Reppert, R. Podila, A. M. Rao, and R. R. Rice. Binary III-V semiconductor core optical fiber. Opt. Express, 18:4972, 2010.

[18] A. C. Peacock, J. R. Sparks, and N. Healy. Semiconductor optical fibres: progress and opportunities. Laser Photon. Rev., 8:53, 2014.

[19] H. K. Tyagi, M. A. Schmidt, L. Prill Sempere, and P. St. J. Russell. Optical properties of photonic crystal fiber with integral micron-sized Ge wire. Opt. Express, 16:17227, 2008.

[20] B. Scott, W. Ke, and G. Pickrell. Fabrication of n-type silicon optical fibers. Photon. Technol. Lett., 21:1798, 2009.

[21] E. Nordstrand, A. Dibbs, A. Eraker, and U. J. Gibson. Alkaline oxide interface modifiers for silicon fiber production. Opt. Mater. Express, 3:651, 2013.

[22] C. Hou, X. Jia, L. Wei, S.-C. Tan, X. Zhao, J. Joannopoulos, and Y. Fink. Crystalline silicon core fibres from aluminium core preforms. Nat. Comm., 6:6248, 2015.

[23] C. Lin, J. Chen, and L. Wang. High-Q Si microsphere resonators fabricated from Si-cored fibers for WGMs excitation. IEEE Photon Technol. Lett., 27:1355, 2015.

[24] G. Tang, Q. Qian, X. Wen, X. Chen, W. Liu, M. Sun, and Z. Yang. Reactive molten core fabrication of glass-clad $\mathrm{Se}_{0.8} \mathrm{Te}_{0.2}$ semiconductor core optical fibers. Opt. Express, 23:23624-23633, 2015.

[25] P. J. Roberts, F. Couny, H. Sabert, B. J. Mangan, D. P. Williams, L. Farr, M. W. Mason, A. Tomlinson, T. A. Birks, J. C. Knight, and P. St. J. Russell. Ultimate low loss of hollow-core photonic crystal fibres. Opt. Express, 13:236, 2005.

[26] N. Healy, L. Lagonigro, J. R. Sparks, S. Boden, P. J. A. Sazio, J. V. Badding, and A. C. Peacock. Polycrystalline silicon optical fibers with atomically smooth surfaces. Opt. Lett., 36:2480, 2011.

[27] N. F. Baril, R. He, T. D. Day, J. R. Sparks, B. Keshavarzi, M. Krishnamurthi, A. Borhan, V. Gopalan, A. C. Peacock, N. Healy, P. J. A. Sazio, and J. V. Badding. Low loss silicon fibers for photonic applications. J. Am. Chem. Soc., 134:19, 2011.

[28] J. Sparks, R. He, N. Healy, M. Krishnamurthi, A. C. Peacock, P. J. A. Sazio, V. Gopalan, and J. V. Badding. Zinc selenide optical fibers. Adv. Mater., 23:1647, 2011.

[29] R. He, P. J. A. Sazio, A. C. Peacock, N. Healy, J. R. Sparks, M. Krishnamurthy, V. Gopalan, and J. V Badding. Integration of gigahertz-bandwidth semiconductor devices inside microstructured optical fibres. Nat. Photon., 6:174, 2012.

[30] J. Ballato and E. Snitzer. Fabrication of fibers with high rare-earth concentrations for faraday isolator applications. Appl. Opt., 34:6848, 1995.

[31] J. Ballato, T. Hawkins, P. Foy, S. Morris, N. K. Hon, B. Jalali, and R. Rice. Silica-clad crystalline germanium core optical fibers. Opt. Lett., 36:687, 2011.

[32] B. L. Scott and G. R. Pickrell. Silicon optical fiber diameter dependent grain size. J. J. Cryst. Growth, 371:134, 2013.

[33] S. Morris, T. Hawkins, P. Foy, C. McMillen, J. Fan, L. Zhu, R. Stolen, R. Rice, and J. Ballato. Reactive molten core fabrication of silicon optical fiber. Opt. Mater. Express, 1:1141, 2011.

[34] S. Morris, T. Hawkins, P. Foy, J. Ballato, S. W. Martin, and R. Rice. Cladding glass development for semiconductor core optical fibres. Int. J. Appl. Glass. Sci., 3:144, 2012.

[35] R. Soref. The past, present, and future of silicon photonics. IEEE J. Quantum Electron., 12:1678, 2006.

[36] R. Soref and J. P. Lorenzo. Single-crystal silicon: A new material for $1.3 \mu \mathrm{m}$ and $1.6 \mu \mathrm{m}$ integratedoptical components. Electron. Lett., 21:953, 1985.

[37] L. Lagonigro, N. Healy, J. R. Sparks, N. F. Baril, P. J. A. Sazio, J. V. Badding, and A. C. Peacock. Low loss silicon fibers for photonic applications. Appl. Phys. Lett., 96:041105, 2010.

[38] N. K. Hon, R. Soref, and B. Jalali. The third-order nonlinear optical coefficients of Si, Ge, and 
$\mathrm{Si}_{1-x} \mathrm{Ge}_{x}$ in the midwave and longwave infrared. J. Appl. Phys., 110:011301, 2011.

[39] S. Mirov, V. Fedorov, I. Moskalev, D. Martyshkin, and C. Kim. Progress in $\mathrm{Cr}^{2+}$ and $\mathrm{Fe}^{2+}$ doped mid-IR laser materials. Laser Photon. Rev., 4:21, 2010.

[40] L. Shen, N. Healy, P. Mehta, T. D. Day, J. R. Sparks, J. V. Badding, and A. C. Peacock. Nonlinear transmission properties of hydrogenated amorphous silicon core fibers towards the mid-infrared regime. Opt. Express, 21:13075, 2013.

[41] N. Gupta, C. McMillen, R. Singh, R. Podila, A. M. Rao, T. Hawkins, P. Foy, S. Morris, R. Rice, K. F. Poole, L. Zhu, and J. Ballato. Annealing of silicon optical fibers. J. Appl. Phys., 110:093107, 2011.

[42] S. Chaudhuri, J. R. Sparks, X. Ji, M. Krishnamurthi, L. Shen, N. Healy, A. C. Peacock, V. Gopalan, and J. V. Badding. Crystalline silicon optical fibers with low optical loss. ACS Photonics, 3:378384, 2016.

[43] N. Healy, S. Mailis, N. M. Bulgakova, P. J. A. Sazio, T. D. Day, J. R. Sparks, H. Y. Cheng, J. V. Badding, and A. C. Peacock. Extreme electronic bandgap modification in laser-crystallized silicon optical fibres. Nat. Mater., 13:1122, 2014.

[44] N. Healy, M. Fokine, Y. Franz, T. Hawkins, M. Jones, J. Ballato, A. C. Peacock, and U. J. Gibson. $\mathrm{CO}_{2}$ laser-induced directional recrystallization to produce single crystal silicon-core optical fibers with low loss. Adv. Optical Mater., DOI: 10.1002/adom.201500784, 2016.

[45] P. Mehta, M. Krishnamurthi, N. Healy, N. F. Baril, J. R. Sparks, P. J. A. Sazio, V. Gopalan, J. V. Badding, and A. C. Peacock. Mid-infrared transmission properties of amorphous germanium optical fibers. Appl. Phys. Lett., 97:071117, 2010.

[46] A. C. Peacock, P. Mehta, P. Horak, and N. Healy. Nonlinear pulse dynamics in multimode silicon core optical fibers. Opt. Lett., 37:3351, 2012.

[47] A. C. Turner, C. Manolatou, B. S. Schmidt, M. Lipson, M. A. Foster, J. E. Sharping, and A. L. Gaeta. Tailored anomalous group-velocity dispersion in silicon channel waveguides. Opt. Express, 14:4357, 2006.

[48] J. C. Knight, T. A. Birks, R. F. Cregan, P. St. J. Russell, and J.-P. de Sandro. Large mode area photonic crystal fibre. Electron. Lett., 34:1347, 1998.

[49] N. Healy, J. R. Sparks, M. N. Petrovich, P. J. A. Sazio, J. V. Badding, and A. C. Peacock. Large mode area silicon microstructured fiber with robust dual mode guidance. Opt. Express, $17: 18076,2009$.

[50] N. Vukovic, N. Healy, and A. C. Peacock. Guiding properties of large mode area silicon microstructured fibers: a route to effective single mode operation. J. Opt. Soc. Am. B, 28:1529, 2011.

[51] J. R. Sparks, R. He, N. Healy, S. Chaudhuri, T. C. Fitzgibbons, A. C. Peacock, P. J. A. Sazio, and J. V. Badding. Conformal coating by high pressure chemical deposition for patterned microwires of II-VI semiconductors. Adv. Funct. Mat., 23:1647, 2013.

[52] T. A. Birks, J. C. Knight, and T. E. Dimmick. High-resolution measurement of the fiber diameter variations using whispering gallery modes and no optical alignment. IEEE Photon. Technol. Lett., 12:182, 2000.

[53] N. Vukovic, N. Healy, P. Horak, J. R. Sparks, P. J. A. Sazio, J. V. Badding, and A. C. Peacock. Ultra-smooth microcylindrical resonators fabricated from the silicon optical fiber platform. Appl. Phy. Lett., 99:031117, 2011.

[54] J. M. Shainline, G. Fernandes, Z. Liu, and J. Xu. Broad tuning of whispering-gallery modes in silicon microdisks. Opt. Express, 18:14345, 2010.

[55] N. Vukovic, N. Healy, J. R. Sparks, J. V. Badding, P. Horak, and A. C. Peacock. Tunable continuous wave emission via phase-matched second harmonic generation in a $\mathrm{ZnSe}$ microcylindrical resonator. Sci. Rep., 5:11798, 2015.

[56] R. H. Stolen and A. Ashkin. Optical Kerr effect in glass waveguide. Appl. Phys. Lett., 22:294, 1973.

[57] J. K. Ranka, R. S. Windeler, and A. J. Stentz. Visible continuum generation in air-silica 
microstructure optical fibers with anomalous dispersion at 800nm. Opt. Lett., 25:25, 2000.

[58] P. Mehta, N. Healy, N. F. Baril, P. J. A. Sazio, J. V. Badding, and A. C. Peacock. Nonlinear transmission properties of hydrogenated amorphous silicon core optical fibers. Opt. Express, 18:16826, 2010.

[59] P. Mehta, N. Healy, J. R. Sparks, T. D. Day, P. J. A. Sazio, J. V. Badding, and A. C. Peacock. All-optical modulation using two-photon absorption in silicon core optical fibers. Opt. Express, 19:19078, 2011.

[60] X. Sang, E.-K. Tien, and O. Boyraz. Applications of two-photon absorption in silicon. J. Optoelectron. Adv. Mater., 11:15, 2008.

[61] M. Dinu. Dispersion of phonon-assisted nonresonant third-order nonlinearities. IEEE J. Sel. Top. Quant. Electron., 39:1498, 2003.

[62] P. Mehta, N. Healy, T. D. Day, J. V. Badding, and A. C. Peacock. Ultrafast wavelength conversion via cross-phase modulation in hydrogenated amorphous silicon optical fibers. Opt. Express, 20:26110, 2012.

[63] L. Shen, N. Healy, L. Xu, H. Y. Cheng, T. D. Day, J. H. V. Price, J. V. Badding, and A. C. Peacock. Four-wave mixing and octave-spanning supercontinuum generation in a small core hydrogenated amorphous silicon fiber pumped in the mid-infrared. Opt. Lett., 39:5721, 2014.

[64] R. Dekker, A. Driessen, T. Wahlbrink, C. Moormann, J. Niehusmann, and M. Först. Ultrafast Kerr-induced all-optical wavelength conversion in silicon waveguides using $1.55 \mu \mathrm{m}$ femtosecond pulses. Opt. Express, 14:8336, 2006.

[65] U. D. Dave, S. Uvin, B. Kuyken, S. Selvaraja F. Leo, and G. Roelkens. Telecom to mid-infrared spanning supercontinuum generation in hydrogenated amorphous silicon waveguides using a thulium doped fiber laser pump source. Opt. Express, 21:32032, 2013.

[66] N. Vukovic, N. Healy, F. H. Suhailin, P. Mehta, T. D. Day, J. V. Badding, and A. C. Peacock. Ultrafast optical control using the Kerr nonlinearity in hydrogenated amorphous silicon microcylindrical resonators. Sci. Rep., 3:2885, 2013.

[67] F. H. Suhailin, L. Shen, N. Healy, L. Xiao, M. Jones, T. Hawkins, J. Ballato, U. J. Gibson, and A. C. Peacock. Tapered polysilicon core fibers for nonlinear photonics. Opt. Lett., 41:1360, 2016.

[68] A. M. Armani, R. P. Kulkarni, S. E. Fraser, R. C. Flagan, and K. J. Vahala. Label-free, singlemolecule detection with optical micro cavities. Science, 317:783, 2007.

[69] O. Boyraz and B. Jalali. Demonstration of a silicon Raman laser. Opt. Express, 12:5269, 2004.

[70] L. Shi, L. A. Sordillo, A. Rodriguez-Contrerar, and R. Alfano. Transmission in near-infrared optical windows for deep brain imaging. J. Biophotonics, 9:38, 2015.

[71] G. Clemens, B. Bird, M. Weida, J. Rowlette, and M. J. Baker. Quantum cascade laser-based midinfrared spectrochemical imaging of tissues and biofluids. Spectroscopy Europe, 26:14, 2014.

[72] J. S. Sanghera and I. D. Aggarwal. Active and passive chalcogenide glass optical fibers for IR applications: a review. J. Non-Cryst. Solids, 256:6, 1999.

[73] J. A. Harrington. A review of IR transmitting, hollow waveguides. Fiber Integrated Opt., 19:211, 2000 .

[74] T. M. Monro and H. Ebendorff-Heidepriem. Progress in microstructured optical fibers. Annu. Rev. Mater. Res., 36:467, 2006.

[75] G. Tao, H. Ebendorff-Heidepriem, A. M. Stolyarov, S. Danto, J. V. Badding, Y. Fink, J. Ballato, and A. F. Abouraddy. Infrared fibers. Adv. Opt. Photon., 7:379, 2015.

[76] R. R. Gattass, R. Thapa, F. H. Kung, L. E. Busse, L. Brandon Shaw, and J. S. Sanghera. Review of infrared fiber-based components. Appl. Opt., 54:F25, 2015. 